\title{
THEOREMS ASSOCIATED WITH THE RIESZ AND THE DIRICHLET'S SERIES METHODS OF SUMMATION
}

\author{
H. L. GARABEDIAN
}

1. Introduction. It is the object of this paper to establish several theorems suggested by a certain theorem due to Hardy.* Hardy's theorem need not be stated here since it is a special case of Theorem 1 of this paper.

We are concerned with the infinite series

$$
\sum_{n=1}^{\infty} u_{n}=u_{1}+u_{2}+\cdots,
$$

where we define $s_{n}=u_{1}+u_{2}+\cdots+u_{n}$.

We list the several different methods of summation with which this account is occupied.

A. The Riesz definition. $\dagger$ We write $C_{\lambda^{r}}(\omega)=\sum_{\lambda_{n}<\omega}\left(\omega-\lambda_{n}\right)^{r} u_{n},(r>0)$, where $\left\{\lambda_{n}\right\}$ is a sequence of real increasing numbers whose limit is infinite and such that $\lambda_{1} \geqq 0$. If $\omega^{-r} C_{\lambda}^{r}(\omega) \rightarrow U$ as $\omega \rightarrow \infty$, the series (1.1) is said to be summable $(\lambda, r)$ to the sum $U$. If in the general definition we put $r=1, \omega=\lambda_{n}$, we obtain

$$
\left(\mu_{1} s_{1}+\mu_{2} s_{2}+\cdots+\mu_{n-1} s_{n-1}\right) / \lambda_{n}
$$

where $\mu_{i}=\lambda_{i+1}-\lambda_{i}$ and $\lambda_{n}=\mu_{1}+\mu_{2}+\cdots+\mu_{n-1}, \lambda_{1}=0$. We refer to means of the type (1.2) as Riesz means, as distinguished from the Riesz typical means of the general definition, and designate them henceforth by the symbol $(\lambda, 1)$. This is the natural generalization of Cesàro's first mean which suggests itself in the attachment of varying weights to the successive partial sums $s_{\nu}$.

B. The Dirichlet's series definitions. $\ddagger$ A series (1.1) is said to be summable by the Dirichlet's series method provided that

$$
\lim _{s \rightarrow 0} \sum_{n=1}^{\infty} u_{n} e^{-\nu_{n} s}
$$

exists, where $\left\{\nu_{n}\right\}$ is a sequence of positive increasing real numbers whose limit is infinite, and where the Dirichlet's series converges when $\Re(s)>0, s$ being restricted to this half plane.

* G. H. Hardy, Proceedings of the London Mathematical Society, (2), vol. 8 (1910), pp. 301-320, p. 311.

$\dagger$ M. Riesz, Comptes Rendus, vol. 149 (1909), pp. 909-912.

¥ See, for example, H. L. Garabedian, Annals of Mathematics, (2), vol. 32 (1931), pp. 83-106, p. 85 . 
2. Extensions of Hardy's theorem. We propose to establish the following theorems.

THEOREM 1. Suppose that $\lim _{n \rightarrow \infty} s_{n} e^{-\nu_{n s}}=0$ for all values of $s$ for which $\Re(s)>0$, that the series (1.1) is summable $(\lambda, 1)$ to the sum $U$, and that $\nu_{n}$ is a logarithmico-exponential* function of $\lambda_{n}$ such that $\nu_{n}=O\left(\lambda_{n}^{\Delta}\right)$, where $\Delta$ is any positive constant. Then, the series $\sum u_{n} e^{-\nu_{n} s}$ converges for $\Re(s)>0$ and

$$
\lim _{s \rightarrow 0} \sum_{n=1}^{\infty} u_{n} e^{-\nu_{n} s}=U
$$

TheOREM 2. The Dirichlet's series definitions of summability include $(\lambda, 1)$ summability provided that $\nu_{n}$ is a logarithmico-exponential function of $\lambda_{n}$ which tends to infinity with $n$ but not as slowly as $\log n$ nor faster than $\lambda_{n}{ }^{\Delta}$, where $\Delta$ is any positive constant however large.

We shall understand in both theorems that $s=\sigma+i \tau$ approaches zero over a point set lying within an angle with vertex at the origin such that

$$
|\operatorname{am} s| \leqq a<\pi / 2
$$

Theorem 1 reduces to Hardy's theorem for the case that $\nu_{n}=\lambda_{n}$. We observe further that the restriction that $\nu_{n+1}-\nu_{n} \rightarrow 0$ as $n \rightarrow \infty$ of Hardy's theorem has been eliminated in Theorem 1.

Theorem 2 eliminates the initial restriction of Theorem 1 and thus affords a regulation inclusion theorem. Theorem 2 recalls the following theorem previously established by the author. $\dagger$

THEOREM 3. The Dirichlet's series definitions of summability include $(C, r)$ summability provided that $\nu_{n}$ is a logarithmico-exponential function of $n$ which tends to infinity with $n$ but not as slowly as $\log n$ nor faster than $n^{\Delta}$, where $\Delta$ is any positive constant however large.

This theorem is at once slightly more general and slightly less general than Theorem 2. To understand this apparent contradiction we need to make use of the following theorem due to Hardy. $\ddagger$

TheOREM 4. If the series (1.1) is summable $(\lambda, r)$ to the sum $U$, and if $\nu_{n}$ is a logarithmico-exponential function of $\lambda_{n}$ such that $\nu_{n}=O\left(\lambda_{n}{ }^{\Delta}\right)$, where $\Delta$ is any positive constant, then the series (1.1) is summable $(\nu, r)$ to the sum $U$.

* Hardy, Proceedings of the London Mathematical Society, (2), vol. 15 (1916), pp. 72-88, p. 75 .

$\dagger$ Loc. cit., footnote 3, p. 105.

$\ddagger$ Loc. cit., footnote 4, p. 72. 
Some examples of the equivalence and inclusion* relations implied by this theorem are

$$
\begin{aligned}
\left(\log _{3} n, 1\right) & \supset\left(\log _{2} n, 1\right) \supset(\log n, 1) \approx\left([\log n]^{2}, 1\right) \supset(n, 1) \\
=(C, 1) \approx\left(n^{r}, 1\right) \approx(n \log n, 1) \supset\left(k^{n}, 1\right) & , \quad r>0, k>1 .
\end{aligned}
$$

Incidentally, summability $\left(k^{n}, 1\right)$, for $k>1$, is equivalent to convergence. $\dagger$

It is also pertinent to this discussion that none of the $(\lambda, 1)$ methods can include summability $(C, r)$ for $r>1$. $\ddagger$ On the other hand, it follows from an example given by Hardy $\S$ that summability $(C, r)$, for $r$ arbitrarily large, cannot include summability $(\log n, 1)$.

It follows as a result of Theorem 3 that all of the Dirichlet's series methods of summation which include $(C, r)$ summability include all of the $(\lambda, 1)$ methods equivalent to $(C, 1)$ summability. Theorem 2 involves a wider class of $(\lambda, 1)$ methods than Theorem 3 but implies nothing as to $(C, r)$ summability for $r>1$.

The particular method of Riesz means, summability $(\log n, 1)$, has been designated as logarithmic summability, $\|$ and has been the source of a number of investigations relating to the summability and convergence of slowly oscillating series. Thus, it is of especial interest that Theorem 2 affords a means of exhibiting a class of Dirichlet's series methods of summation which include summability $(\log n, 1)$. This result is expressed in the following corollary to Theorem 2.

COROLlary. The Dirichlet's series methods of summation include summability $(\log n, 1)$ provided that $\nu_{n}$ is a logarithmico-exponential function of $n$ which tends to infinity with $n$ but not as slowly as $\log n$ nor faster than $(\log n)^{\Delta}$, where $\Delta$ is any positive constant however large.

3. Proofs of Theorems 1 and 2. The proofs of Theorems 1 and 2, which for the present are carried simultaneously, are based on several theorems due to Hardy and Riesz to which we shall refer as the occasion arises.

* We understand $A \subset B$ to mean that every series summable by a method $\mathrm{A}$ is also summable by a method $B$ to the same limit, or that the method $B$ includes the method A. Further, we interpret $A \approx B$ to mean that the two methods are equivalent, that is, each method includes the other.

$\dagger$ Hardy and Riesz, The General Theory of Dirichlet's Series, Cambridge Tracts in Mathematics and Mathematical Physics, no. 18, p. 35.

$\ddagger$ H. L. Garabedian and W. C. Randels, Duke Mathematical Journal, vol. 4 (1938), pp. 529-533, p. 531.

$\S$ Loc. cit., footnote 1, p. 310.

|l Hardy, loc. cit., footnote 4. 
Since by assumption

$$
\sum_{n=1}^{\infty} u_{n} e^{-\nu_{n} s}
$$

is summable $(\lambda, 1)$, for $s=0$, to the sum $U$, it is also summable $(\nu, 1)$, for $s=0$, to the same value. This statement follows from Theorem 4 .

Since $(1.1)$ is summable $(\nu, 1),(3.1)$ is uniformly summable $(\nu, 1)$ throughout the angle a to the sum $f(s)$. This statement is due to Hardy and Riesz.*

Inasmuch as $(3.1)$ is summable $(\nu, 1)$ to the sum $U$, for $s=0$, and since $(3.1)$ is uniformly summable $(\nu, 1)$ to the sum $f(s)$ throughout the angle $a, f(s) \rightarrow U$ as $s \rightarrow 0$ along any path lying entirely within the angle $a$. This follows from another theorem due to Hardy and Riesz. $\dagger$

If the hypotheses of Theorem 1 obtain and if $\left\{\nu_{n+1}-\nu_{n}\right\}$ is a null sequence, then the series (3.1) converges for $\Re(s)>0$. $\ddagger$ The extra restriction will be eliminated by an argument given below. Now, the series (3.1) converges to the value $f(s)$ to which it is also summable $(\nu, 1)$. It follows that the value approached as $s \rightarrow 0$ must be the same in both cases. Thus, with the restrictions on $\nu_{n}$ which have been stated,

$$
\lim _{s \rightarrow 0} \sum_{n=1}^{\infty} u_{n} e^{-v_{n} s}=U
$$

This establishes Theorem 1.

The abscissa of convergence of a Dirichlet's series is given by the formula $\S$

$$
\sigma_{0}=\limsup _{n \rightarrow \infty}\left(\log \left|s_{n}\right|\right) / \nu_{n}
$$

Now, it is known that if the series $(1.1)$ is summable $(\nu, 1)$ to the value $U$, then $\|$

$$
\varsigma_{n}=o\left(\nu_{n+1} /\left(\nu_{n+1}-\nu_{n}\right)\right)+U .
$$

Thus, it is easily verified that if the series (1.1) is summable $(\nu, 1)$, then $s_{n}=o\left(n^{2}\right)$. Accordingly, we have in this case

* Hardy and Riesz, loc. cit., footnote 8, Theorem 23.

$\dagger$ Hardy and Riesz, loc. cit., footnote 8, Theorem 28.

$\ddagger$ Hardy, loc. cit., footnote 1, p. 311. It is assumed in Hardy's theorem that $\lambda_{n+1} / \lambda_{n} \rightarrow 1$. However, we note that, of the $(\lambda, 1)$ methods listed in $(2.1)$, this restriction eliminates only the trivial case for which $\lambda_{n}=k^{n},(k>1)$.

$\S$ Hardy and Riesz, loc. cit., footnote 8, Theorem 7.

|| Hardy and Riesz, loc. cit., footnote 8, Theorem 21. 


$$
\sigma_{0} \leqq \limsup _{n \rightarrow \infty}(2 \log n) / \nu_{n}
$$

In order that $\sigma_{0} \leqq 0$, in which case (3.1) will converge in the right half of the $s$-plane, it is sufficient that $\nu_{n}$ tend to infinity faster than $\log n$. The argument used to complete the proof of Theorem 2 is the same as the one used above in connection with Theorem 1.

Notice that if $\left\{\nu_{n+1}-\nu_{n}\right\}$ is not a null sequence, then $\nu_{n}$ tends to infinity faster than $\log n$. This eliminates the extra restriction used in the proof of Theorem 1.

\section{NoRThWESTERN UNIVERSITY}

\section{ON CERTAIN IDEALS OF DIFFERENTIAL POLYNOMIALS*}

\section{J. F. RITT AND E. R. KOLCHIN}

Introduction. Let $\Sigma$ be an ideal of differential polynomials in the unknowns $y_{1}, \cdots, y_{n}$. If the manifold of $\Sigma$ is composed of $s$ manifolds $\mathfrak{M}_{1}, \cdots, \mathfrak{M}_{s}$ not necessarily irreducible, none of which has a solution in common with any other, $\Sigma$ has a unique representation as the product of $s$ ideals $\Sigma_{1}, \cdots, \Sigma_{s}$ whose manifolds are, respectively, the $\mathfrak{M}_{i} \bullet$

Most of the present note is concerned with decompositions of the foregoing type and considers the case in which one of the $\mathfrak{M}_{i}$, say $\mathfrak{M}_{1}$, is composed of a single solution, that is, of a set of functions $\bar{y}_{1}, \cdots, \bar{y}_{n}$ contained in the underlying field. We shall examine, for this special case, the structure of the ideal $\Sigma_{1}$. Details will be given only for the case of a single unknown; the extensions to several unknowns are too obvious to require explicit mention. It will suffice, furthermore, to treat the case in which $\mathfrak{M}_{1}$ is composed of the solution $y=0$.

In $\$ 9$, we consider a problem closely related to the theorem of decomposition stated above.

1. On the structure of $\Sigma_{1}$. Let $\Sigma$ be an ideal of forms in the unknown $y$. Let $y=0$ be an essential irreducible manifold for $\Sigma$. Let $\Sigma$ be the product of $\Sigma_{1}$ and $\Sigma_{2}$ where $\Sigma_{1}$ has $y=0$ as its manifold and $\Sigma_{2}$ does not admit $y=0$ as a solution. Let $p$ be a positive integer such that $y^{p}$ is contained in $\Sigma_{1}$.

* Presented to the Society, September 8, 1939.

$\dagger$ Proceedings of the National Academy of Sciences, vol. 25 (1939), p. 90. Product is defined in the expected way. That the intersection of the $\Sigma_{i}$ is identical with their product follows immediately from the fact that the $\Sigma_{i}$, considered as algebraic ideals, are paarweise teilerfremd. See van der Waerden, Moderne Algebra, vol. 2, p. 46. 